\title{
Macrofauna invertebrada do solo sob diferentes sistemas de produção em Latossolo da Região do Cerrado
}

\author{
Rogério Ferreira da Silva ${ }^{(1)}$, Adriana Maria de Aquino ${ }^{(2)}$, Fábio Martins Mercante ${ }^{(3)}$ e Maria de Fátima Guimarães $^{(1)}$ \\ (1)Universidade Estadual de Londrina, Centro de Ciências Agrárias, Caixa Postal 6001, CEP 86051-990 Londrina, PR. E-mail: \\ rogerio@cpao.embrapa.br, mfatima@uel.br (2)Embrapa Agrobiologia, CEP 23890-000 Seropédica, RJ. E-mail: adriana@cnpab.embrapa.br \\ (3)Embrapa Agropecuária Oeste, Caixa Postal 661, CEP 79804-970 Dourados, MS. E-mail: mercante@cpao.embrapa.br
}

\begin{abstract}
Resumo - O objetivo deste trabalho foi quantificar a densidade e diversidade de grupos da comunidade de macrofauna invertebrada do solo sob diferentes sistemas de produção, bem como analisar a similaridade entre os sistemas avaliados. O trabalho foi conduzido no Município de Dourados, MS, em Latossolo Vermelho distroférrico típico, sob os seguintes sistemas: sistema convencional (SC), sistema plantio direto (SPD), sistema integração lavoura/pecuária (SILP), pastagem contínua (PC) e vegetação nativa. As amostragens foram realizadas em três safras de verão e de inverno. Os valores de densidade total da comunidade de macrofauna invertebrada de solo, nos sistemas SPD, SILP e PC, foram similares, no entanto, o SPD apresentou a maior diversidade de grupos. Na safra de verão, observou-se similaridade entre os sistemas SPD e SILP e entre o SC e PC; e na safra de inverno, a similaridade foi entre os sistemas SPD, SILP e PC.
\end{abstract}

Termos para indexação: manejo do solo, similaridade, densidade, diversidade.

\section{Soil invertebrate macrofauna under different production systems in a Hapludox in the Cerrado Region}

\begin{abstract}
The objective of this work was to quantify the density and diversity of soil invertebrate macrofauna community, in different production systems, and also to evaluate the similarity among them. The study was carried out in the Municipality of Dourados, MS, Brazil, in a Typic Hapludox under the following systems: conventional system (CS), no-tillage system (NTS), crop/livestock integration system (CLIS), continuous pasture (CP) and natural system. Samples were collected during summer and winter cropping seasons. Total density and diversity of the soil invertebrate macrofauna community in the NTS, CLIS, and CP systems were similar. However, NTS showed the highest diversity. In summer, similarity was observed between NTS and CLIS, and between CS and CP, with regard to the soil invertebrate macrofauna community. In winter, similarity was among NTS, CLIS and CP.
\end{abstract}

Index terms: soil management, similarity, density, diversity.

\section{Introdução}

O Cerrado foi incorporado, nas últimas quatro décadas, ao cenário mundial da produção de grãos e carne bovina. O resultado foi um ecossistema artificial e instável, com elevada simplificação florística e faunística, que requer intervenção humana constante na forma de fluxos de energia, como fertilizantes, corretivos, agrotóxicos e reguladores de crescimento. Como resultado desse processo de desenvolvimento, esta região atualmente apresenta uma área plantada de 13,5 milhões de hectares, com culturas anuais, e 45 milhões de hectares de pastagem (Embrapa, 1998). Estima-se que 50\% dos solos sob culturas anuais e $80 \%$ sob pastagem en- contram-se com certo grau de degradação (Kluthcouski \& Aidar, 2003).

Este cenário de degradação serviu de estímulo à adoção de práticas de produção agropecuária mais conservacionistas, em que se destacam o sistema plantio direto, com rotação de culturas, e o sistema integração lavoura/pecuária, trazendo grandes mudanças no controle de erosão e na sustentabilidade da atividade agrícola. Estes sistemas contribuem para a manutenção de adequada umidade, temperatura e matéria orgânica do solo, o que beneficia a flora e fauna do solo, e induz maior ocorrência de organismos benéficos (Campanhola, 2002). 
Entre os organismos que constituem a fauna do solo, a macrofauna edáfica compreende os maiores invertebrados (organismos com mais que $10 \mathrm{~mm}$ de comprimento ou com mais de $2 \mathrm{~mm}$ de diâmetro corporal), como minhocas, coleópteros em estado larval e adulto, centopéias, cupins, formigas, piolhos de cobra (milipéias), tatuzinhos e aracnídeos (Wolters, 2000; Lavelle \& Spain, 2001).

A macrofauna invertebrada do solo desempenha um papel chave no funcionamento do ecossistema, pois ocupa diversos níveis tróficos dentro da cadeia alimentar do solo e afeta a produção primária de maneira direta e indireta. Altera, por exemplo, as populações e atividade de microrganismos responsáveis pelos processos de mineralização e humificação e, em conseqüência, exerce influência sobre o ciclo de matéria orgânica e a disponibilidade de nutrientes assimiláveis pelas plantas (Decäens et al., 2003). A macrofauna pode ser também vetora de microrganismos simbióticos das plantas, como fixadores de nitrogênio e fungos micorrízicos, e é capaz de digerir, de maneira seletiva, microrganismos patogênicos (Brown, 1995).

Alguns organismos da macrofauna, principalmente os térmitas, as formigas, as minhocas e larvas de coleópteros, são denominados "engenheiros do ecossistema”, pois suas atividades levam à criação de estruturas biogênicas (galerias, ninhos, câmaras e bolotas fecais), que modificam as propriedades físicas dos solos onde vivem e a disponibilidade de recursos para outros organismos (Wolters, 2000). Por meio de suas ações mecânicas no solo, a macrofauna contribui na formação de agregados estáveis, que podem proteger parte da matéria orgânica de uma mineralização rápida e que constituem, também, uma reserva de nutrientes potencialmente disponível para as plantas (Lavelle \& Spain, 2001; Decäens et al., 2003).

Apesar da relevância da macrofauna edáfica para o funcionamento dos ecossistemas, poucos estudos têm sido realizados, especialmente em solos do Cerrado, que avaliam os efeitos das práticas de manejos sobre os organismos da macrofauna, já que a maioria dos trabalhos que envolve a biologia do solo tem dedicado atenção à microflora e microfauna. Os organismos da macrofauna respondem às diversas intervenções antrópicas realizadas no meio ambiente (Lavelle \& Spain, 2001). Portanto, a densidade e diversidade desses organismos, assim como a presença de determinados grupos específicos em um sistema, podem ser usadas como indicadores da qualidade dos solos (Chaussod, 1996; Tian et al., 1997; Paoletti, 1999; Barros et al., 2003).

O presente trabalho teve como objetivo quantificar a densidade e diversidade de grupos da comunidade de macrofauna invertebrada do solo sob diferentes sistemas de produção, bem como utilizar esses parâmetros para avaliar a similaridade entre os agrecossistemas estudados.

\section{Material e Métodos}

O estudo foi realizado no período de 2000 a 2003, num experimento estabelecido em 1995, na Embrapa Agropecuária Oeste, em Dourados, MS (22 ${ }^{\circ} 14$ ' S e $54^{\circ} 49^{\prime}$ W), em Latossolo Vermelho distroférrico típico, $70 \%$ de argila. O clima da região, segundo a classificação de Köppen, é Aw, com estação quente e chuvosa no verão e moderadamente seca no inverno. Os dados agroclimáticos mensais de precipitação pluvial (mm) e temperatura $\left({ }^{\circ} \mathrm{C}\right)$, durante o período de estudo, encontram-se na Figura 1.

O delineamento experimental adotado foi o de blocos ao acaso, em esquema fatorial (5x6), com cinco sistemas de produção e seis épocas de avaliação, com cinco repetições.

Os tratamentos foram dispostos em faixas, sob quatro sistemas de produção. O sistema convencional (SC), estabelecido em área de 2 ha, consistiu de cultivo de soja (Glycine max Merril) no verão, e de aveia (Avena strigosa Schreb) no outono/inverno; o solo foi preparado com uso de grade de disco, e herbicidas residuais foram aplicados em pré-emergência. O sistema plantio direto (SPD) constituiu-se de faixa subdividida em três partes de 2,8 ha, das quais duas ocupadas com soja e uma com milho, no verão, e com aveia, trigo e nabo forrageiro durante o outono/inverno. As culturas de trigo e aveia foram utilizadas para produção de grãos, enquanto o nabo forrageiro foi utilizado para produção de palha. Nesse sistema, foram empregados herbicidas pósemergentes e outras tecnologias disponíveis para a região, a fim de se obterem elevados rendimentos de grãos e se reduzirem as perdas do sistema. O sistema integrado lavoura/pecuária (SILP) foi estabelecido em faixa subdividida em duas partes de 4 ha, uma ocupada com lavoura e outra com pastagem (Brachiaria decumbens Stapf) - sistema rotacionado a cada dois anos. Na área ocupada com lavoura, a soja conduzida em SPD, no verão, era rotacionada com aveia no inverno, utilizada como pastagem temporária. Finalmente, o sistema de 
pastagem contínua (PC) consistiu de pastagem permanente (B. decumbens), em área de aproximadamente 10 ha. Uma área adjacente de aproximadamente 5 ha, com vegetação nativa do tipo Cerrado, foi incluída no estudo, como referencial da condição original do solo.

Os resultados referentes ao sistema plantio direto e ao sistema integrado lavoura/pecuária correspondem a valores médios das subdivisões das respectivas faixas. Nos sistemas em estudo foram definidos, aleatoriamente, cinco pontos de amostragem eqüidistantes em $30 \mathrm{~m}$, ao longo de um transecto. Em cada ponto, foi amostrado um monólito de solo de $0,25 \times 0,25 \mathrm{~m}$ de largura e $0,3 \mathrm{~m}$ de profundidade, de acordo com as recomendações de Anderson \& Ingram (1993). As amostragens foram feitas em seis épocas: dezembro de 2000; junho de 2001; janeiro e junho de 2002; janeiro e julho de 2003, correspondentes a três safras de verão e três de inverno.

Para avaliar a diversidade de grupos (número de grupos) e a densidade dos indivíduos (indivíduos $\mathrm{m}^{-2}$ ) da comunidade de macrofauna invertebrada, nos sistemas, as amostras de solo foram triadas manualmente, e os organismos encontrados com mais que $10 \mathrm{~mm}$ de comprimento ou com diâmetro corporal superior a $2 \mathrm{~mm}$ foram extraídos e armazenados numa solução de álcool 70\%. Com auxílio de lupa binocular, procedeu-se à identificação e contagem dos organismos dos grandes grupos taxonômicos. Além disso, os organismos foram separados, de acordo com estádio de desenvolvimento, em adultos ou imaturos (larvas).

Em todas as épocas de avaliação, as amostras de solo foram coletadas à profundidade $0-0,3 \mathrm{~m}$, para a caracterização química, de acordo com Embrapa (1997) (Tabela 1).

Os dados de macrofauna obtidos (x), dada a sua heterogeneidade, foram transformados em $\ln (\mathrm{x}+1) \mathrm{e}$, depois, submetidos à análise de variância (teste F). As médias foram comparadas pelo teste de Tukey a 5\% de probabilidade. Calculou-se o coeficiente de correlação entre os dados de densidade total e diversidade de grupos da macrofauna e os de teores de matéria orgânica do solo. Além disso, os dados foram submetidos à análise de agrupamento (cluster analysis), adotando-se o método do vizinho mais distante (complete linkage) a partir da Distância Euclidiana, para descrever a similaridade entre os sistemas estudados. As análises estatísticas foram processadas por meio do programa Statistica (versão 5.0, StatSoft).

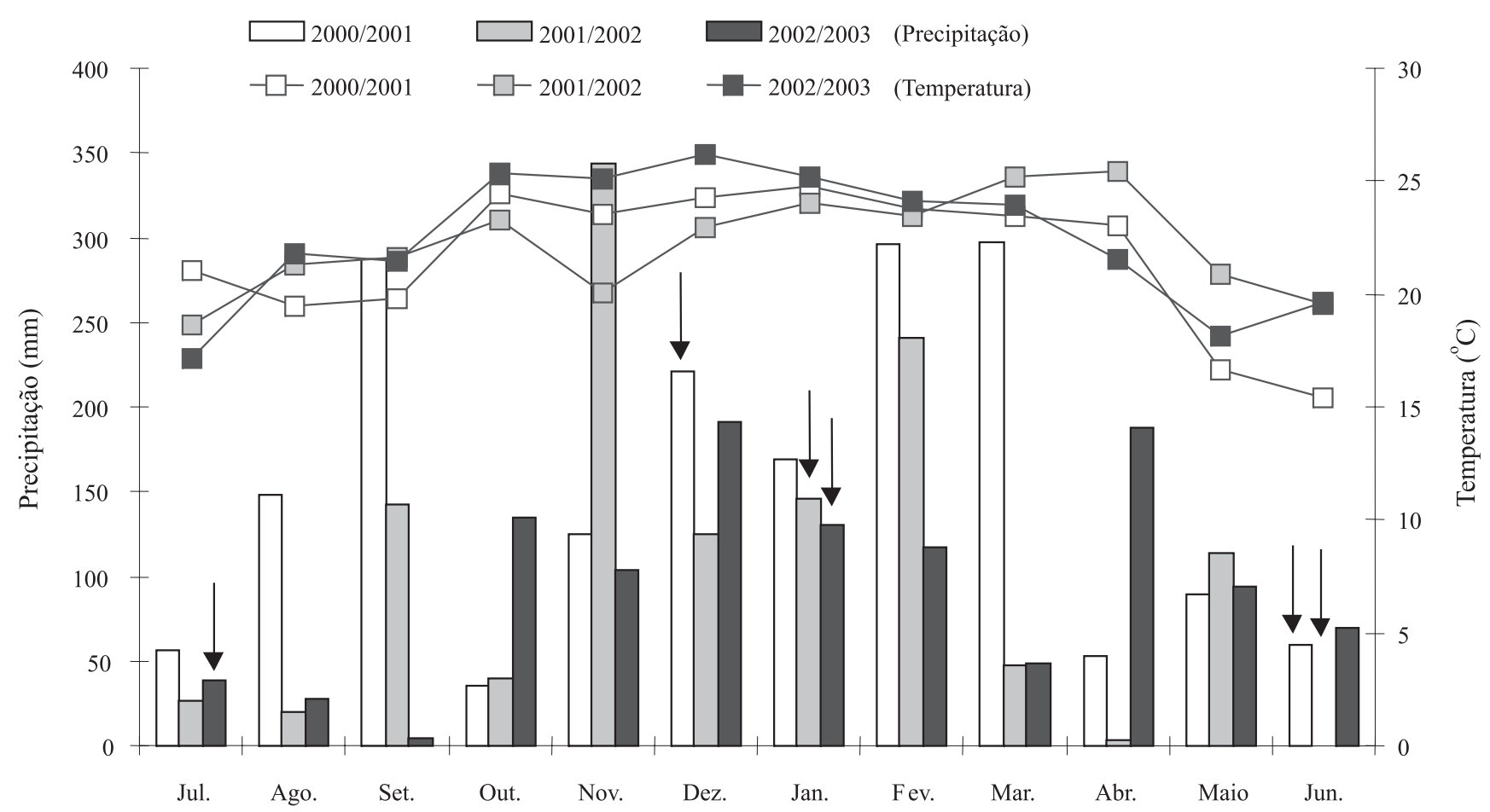

Figura 1. Precipitação pluvial e temperatura média mensal da Estação de Agroclimatologia da Embrapa Agropecuária Oeste, nos anos agrícolas 2000/2001, 2001/2002 e 2002/2003. As setas indicam os meses em que foram realizadas as amostragens da macrofauna edáfica. 


\section{Resultados e Discussão}

A densidade total e a diversidade de grupos da macrofauna edáfica diferiram estatisticamente entre os sistemas (Tabela 2). Entre todos os sistemas, a vegetação nativa apresentou a maior densidade total e diversidade de grupos, por ser um ambiente mais favorável em termos de variedade de micro-habitats e oferta de recursos.

Os sistemas SPD, SILP e PC apresentaram valores de densidade total semelhantes, mas significativamente superiores ao SC (Tabela 2). Os grupos de maior densidade foram: Formicidae em SPD (38\% do total) e Isoptera em SC, SILP e PC (39, 44 e 52\% do total, respectivamente). Foi detectada uma correlação positiva e significativa $(r=0,37 ; \mathrm{p}<0,05)$ entre a matéria orgânica e a densidade total (soma de todos os grupos).

A macrofauna edáfica é altamente sensível a interferências antrópicas; portanto, o tipo de manejo e as pra-

Tabela 1. Caracterização química de solo, à profundidade 0-30 cm, sob diferentes sistemas de produção ${ }^{(1)}$.

\begin{tabular}{|c|c|c|c|c|c|c|c|}
\hline Sistema & $\begin{array}{c}\mathrm{pH} \\
\left(\mathrm{H}_{2} \mathrm{O}\right) \\
\end{array}$ & $\begin{array}{c}\mathrm{P} \\
\left(\mathrm{mg} \mathrm{dm}^{-3}\right)\end{array}$ & K & $\begin{array}{c}\mathrm{Ca} \\
-(\mathrm{cmo} \\
\end{array}$ & $\begin{array}{r}\mathrm{Mg} \\
\left.\mathrm{dm}^{-3}\right) \\
\end{array}$ & Al & $\begin{array}{c}\mathrm{MO} \\
\left(\mathrm{g} \mathrm{kg}^{-1}\right) \\
\end{array}$ \\
\hline $\mathrm{SC}$ & $5,2 d$ & $11,9 \mathrm{~b}$ & $0,4 \mathrm{~b}$ & $3,2 b c$ & $1,2 \mathrm{~d}$ & $1,0 \mathrm{a}$ & $25,8 \mathrm{c}$ \\
\hline SPD & $5,3 \mathrm{c}$ & $17,2 \mathrm{a}$ & $0,4 b$ & $3,8 b$ & $1,7 \mathrm{c}$ & $0,6 \mathrm{~b}$ & $26,2 \mathrm{c}$ \\
\hline SILP & $5,3 \mathrm{c}$ & $8,2 \mathrm{c}$ & $0,2 \mathrm{c}$ & $3,0 \mathrm{c}$ & $1,4 \mathrm{~cd}$ & $0,8 \mathrm{~b}$ & $27,2 \mathrm{c}$ \\
\hline $\mathrm{PC}$ & $5,5 b$ & $3,7 d$ & $0,3 b$ & $3,6 b$ & $2,0 \mathrm{~b}$ & $0,3 \mathrm{c}$ & $30,0 \mathrm{~b}$ \\
\hline $\mathrm{SN}$ & $6,0 \mathrm{a}$ & $1,8 \mathrm{e}$ & $0,5 \mathrm{a}$ & $9,5 \mathrm{a}$ & $2,8 \mathrm{a}$ & $0,1 \mathrm{~d}$ & $49,2 \mathrm{a}$ \\
\hline
\end{tabular}

(1)SC: sistema convencional; SPD: sistema plantio direto; SILP: sistema integrado lavoura/pecuária; PC: pastagem contínua e SN: sistema natural sob vegetação nativa; os dados representam médias de seis épocas de avaliação; médias seguidas de letras diferentes, na mesma coluna, diferem entre si, pelo teste de Tukey a $5 \%$ de probabilidade. ticas culturais determinam a presença e a quantidade nos solos (Didden et al., 1994). A densidade de Arachnida em SPD e Chilopoda em PC foi similar à da vegetação nativa (Tabela 2 ).

Os resultados em SPD corroboraram os de Robertson et al. (1994), Marín et al. (2001), Benito (2002) e Cividanes (2002), de que o SPD possibilita maior densidade de predadores (Arachnida e Chilopoda). Esses artrópodes podem promover o controle de pragas agrícolas, reduzindo o uso de inseticidas em sistemas cultivados (Ekschmitt et al., 1997) e estão relacionados a habitats mais diversificados (Merlim et al., 2005). Em relação aos organismos do grupo Coleoptera (adultos e imaturos), esta similaridade com a vegetação nativa foi observada no SPD (adultos e imaturos), SILP (adultos) e PC (imaturos).

Verificou-se uma maior ocorrência de Heteroptera na área cultivada com pastagem contínua (PC). Entre os organismos encontrados nesse grupo, destaca-se um inseto-praga denominado popularmente de percevejocastanho-da-raiz das pastagens (Scaptocoris spp.) que, conforme Oliveira \& Malaguido (2004), pode ocorrer em várias culturas, principalmente soja e pastagem.

A densidade de minhocas em SILP e PC foi similar à da vegetação nativa, provavelmente em razão da maior densidade de raízes nas pastagens, o que implica em maior conteúdo de matéria orgânica (Tabela 1), que proporciona melhor ambiente para esses organismos (Chan, 2001). Conforme observações de Lavelle (1988), as áreas com pastagens permanentes podem apresentar uma densidade de minhocas três a quatro vezes superior à das áreas ocupadas com lavoura.

Tabela 2. Densidade dos indivíduos (indivíduos $\mathrm{m}^{-2}$ ) e número de grupos da comunidade de macrofauna invertebrada do solo, sob diferentes sistemas de produção ${ }^{(1)}$.

\begin{tabular}{|c|c|c|c|c|c|}
\hline Grupo taxonômico & $\mathrm{SC}$ & SPD & SILP & $\mathrm{PC}$ & $\mathrm{SN}$ \\
\hline Arachnida & $2 \pm 0,9 \mathrm{c}$ & $10 \pm 2,2 \mathrm{ab}$ & $5 \pm 1,0 \mathrm{bc}$ & $6 \pm 1,8 \mathrm{bc}$ & $21 \pm 4,4 a$ \\
\hline Heteroptera & $2 \pm 1,3 \mathrm{c}$ & $6 \pm 2,0 \mathrm{c}$ & $20 \pm 5,4 b$ & $142 \pm 36,8 \mathrm{a}$ & $7 \pm 2,4 \mathrm{c}$ \\
\hline Coleoptera & $19 \pm 4,1 b$ & $37 \pm 5,5 \mathrm{a}$ & $30 \pm 5,1 \mathrm{ab}$ & $26 \pm 7,6 b$ & $123 \pm 81,1 \mathrm{a}$ \\
\hline Chilopoda & $8 \pm 3,5 c$ & $10 \pm 3,0 \mathrm{bc}$ & $11 \pm 2,0 \mathrm{bc}$ & $18 \pm 5,0 \mathrm{a}$ & $50 \pm 11,3 \mathrm{a}$ \\
\hline Oligochaeta (minhocas) & $15 \pm 5,7 \mathrm{c}$ & $57 \pm 12,1 b$ & $77 \pm 26,3 \mathrm{ab}$ & $73 \pm 13,6 \mathrm{ab}$ & $107 \pm 13,1 \mathrm{a}$ \\
\hline Oligochaeta (Enchytraeidae) & $2 \pm 1,3 \mathrm{c}$ & $30 \pm 16,3 b$ & $5 \pm 4,3 c$ & $7 \pm 2,9 \mathrm{c}$ & $1574 \pm 295,4 a$ \\
\hline Formicidae & $147 \pm 42,7 \mathrm{c}$ & $345 \pm 62,0 \mathrm{ab}$ & $307 \pm 59,7 \mathrm{ab}$ & $366 \pm 154,3 \mathrm{ab}$ & $713 \pm 174,5 a$ \\
\hline Isoptera & $188 \pm 84,1 \mathrm{bc}$ & $255 \pm 90,0 \mathrm{ab}$ & $457 \pm 89,8 \mathrm{a}$ & $900 \pm 523,9 a$ & $120 \pm 100,5 c$ \\
\hline Larva de Coleoptera & $70 \pm 13,3 b$ & $100 \pm 18,2 \mathrm{ab}$ & $68 \pm 11,0 \mathrm{~b}$ & $101 \pm 34,3 \mathrm{ab}$ & $146 \pm 24,7 \mathrm{a}$ \\
\hline Outras larvas & $13 \pm 5,4 b$ & $29 \pm 13,1 \mathrm{ab}$ & $22 \pm 11,2 b$ & $33 \pm 22,1 \mathrm{ab}$ & $60 \pm 34,4 a$ \\
\hline Outros grupos de invertebrados & $19 \pm 5,3 \mathrm{c}$ & $35 \pm 6,7 \mathrm{~b}$ & $26 \pm 6,1 \mathrm{~b}$ & $44 \pm 8,6 \mathrm{~b}$ & $185 \pm 19,4 \mathrm{a}$ \\
\hline Total de indivíduos & $484 \pm 106,1 \mathrm{c}$ & $913 \pm 136,2 b$ & $1027 \pm 113,7 b$ & $1715 \pm 605,0 \mathrm{~b}$ & $3106 \pm 388,2 \mathrm{a}$ \\
\hline Número de grupos ${ }^{(2)}$ & $9 \mathrm{~d}$ & $19 \mathrm{~b}$ & $15 \mathrm{c}$ & $15 \mathrm{c}$ & $21 \mathrm{a}$ \\
\hline
\end{tabular}


Em relação ao sistema convencional, verificou-se uma superioridade do SPD, SILP e PC na densidade de minhocas, provavelmente em virtude do maior acúmulo de matéria orgânica nesses sistemas e, principalmente, do tipo de preparo do solo, caracterizado pela ausência de revolvimento.

No sistema sob vegetação nativa, os organismos do grupo Enchytraeidae representaram 51\% da densidade total - significativamente superior aos demais sistemas. Entre os sistemas produtivos, a maior densidade de Enchytraeidae foi observada no SPD. Alguns autores sugerem efeitos antagônicos entre Enchytraeidae e outros grupos de Oligochaeta, e mencionam que na vegetação nativa existe maior oportunidade para esses grupos ocuparem nichos diferentes (Didden et al., 1997; Räty \& Huhta, 2003).

Com relação à densidade de formigas (Formicidae), todos os sistemas foram similares, com exceção do SC, que apresentou a menor densidade. De modo geral, todos os sistemas apresentaram organismos deste grupo em alta densidade, considerados de fundamental importância para os processos de decomposição (Lavelle \& Spain, 2001). Apesar da elevada densidade de formigas nos sistemas cultivados, isso não significa que esse seja o ambiente com a estrutura de comunidade mais complexa. As formigas se adaptam facilmente às condições locais, podendo haver predomínio de uma ou poucas espécies. A ocorrência ampla, associada à variedade de hábitos alimentares, confere a esses organismos o potencial de atuar como eficientes polinizadores, dispersores de sementes, detritívoros e predadores, participando ativamente do equilíbrio dinâmico de agroecossistemas conservacionistas (Lobry de Bruyn, 1999).

No caso dos térmitas (Isoptera), a densidade foi maior no SILP e PC, provavelmente em razão da alta relação $\mathrm{C} / \mathrm{N}$ da matéria orgânica, beneficiando-se da associação com microrganismos (Lavelle \& Spain, 2001), o que permite digerir substratos complexos (Sanchéz \& Reinés, 2001). A diminuição da disponibilidade e da qualidade da pastagem promove um aumento de celulose no sistema, implica maior disponibilidade de alimento para os térmitas e, conseqüentemente, contribui para a sua maior proliferação (Benito et al., 2004).

Alguns organismos adultos ou em forma imatura (larvas) ocorreram eventualmente em alguns sistemas, sob baixa densidade, sendo agrupados como "outros invertebrados" e "outras larvas”, respectivamente.
A vegetação nativa apresentou a maior diversidade de grupos, sendo significativamente superior aos sistemas cultivados (Tabela 2). Entre os sistemas de produção, o SPD apresentou a maior diversidade de grupos, indicando que a rotação de culturas aumenta a diversificação vegetal, a entrada de diferentes tipos de exsudatos e resíduos culturais, e promove melhoria da qualidade de matéria orgânica, o que favorece as condições para o aumento da diversidade de grupos de macrofauna edáfica. No SPD, a maior disponibilidade de energia e nutrientes, associada à existência de novos habitats favoráveis à colonização da macrofauna invertebrada do solo, influencia a densidade e a diversidade de praticamente todos os grupos presentes (Benito, 2002; Merlim et al., 2005).

Esses resultados corroboram os conceitos de Odum (1989), sobre a relação entre estabilidade do sistema e a diversidade de espécies. Para esse autor, em ambientes homogêneos, a densidade tende a ser alta e a diversidade tende a diminuir. Esse aspecto tem sido considerado de fundamental importância para a melhoria da dinâmica da matéria orgânica do solo e favorecimento da ciclagem de nutrientes e do crescimento vegetal (Lavelle \& Spain, 2001).

Observou-se, ainda, que a diversidade de grupos foi semelhante entre SILP e PC, e superior à do SC. Houve correlação positiva e significativa entre o conteúdo de matéria orgânica do solo e a diversidade de grupos da macrofauna, confirmando a sua importância como fonte de alimento para estes organismos.

$\mathrm{Na}$ análise de agrupamento, verificou-se a formação de quatro grupos distintos entre os sistemas (G1, G2, G3 e G4), tanto na safra de verão como na de inverno (Figura 2). Na safra de verão, houve um agrupamento entre o SPD e o SILP, com $88 \%$ de semelhança, e entre o SC e a PC, com 92\% de semelhança. Na safra de inverno, o sistema com PC se agrupou com o SPD e SILP, com $86 \%$ de similaridade. Isso indica que, na PC, o agrupamento possivelmente ocorreu em virtude do efeito da cobertura vegetal, no sentido de favorecer as condições edáficas para a comunidade de macrofauna, principalmente quanto à temperatura e umidade.

A formação do agrupamento G2 (vegetação nativa em dezembro de 2000 e SILP em janeiro de 2003), na safra de verão, pode ter ocorrido em virtude da similaridade da densidade das minhocas e cupins entre os dois sistemas. O agrupamento G1 (PC em junho de 2002), na safra de inverno, possivelmente ocorreu por causa da alta densidade de Isoptera (68\% da densidade total) observada nessa época de avaliação. 

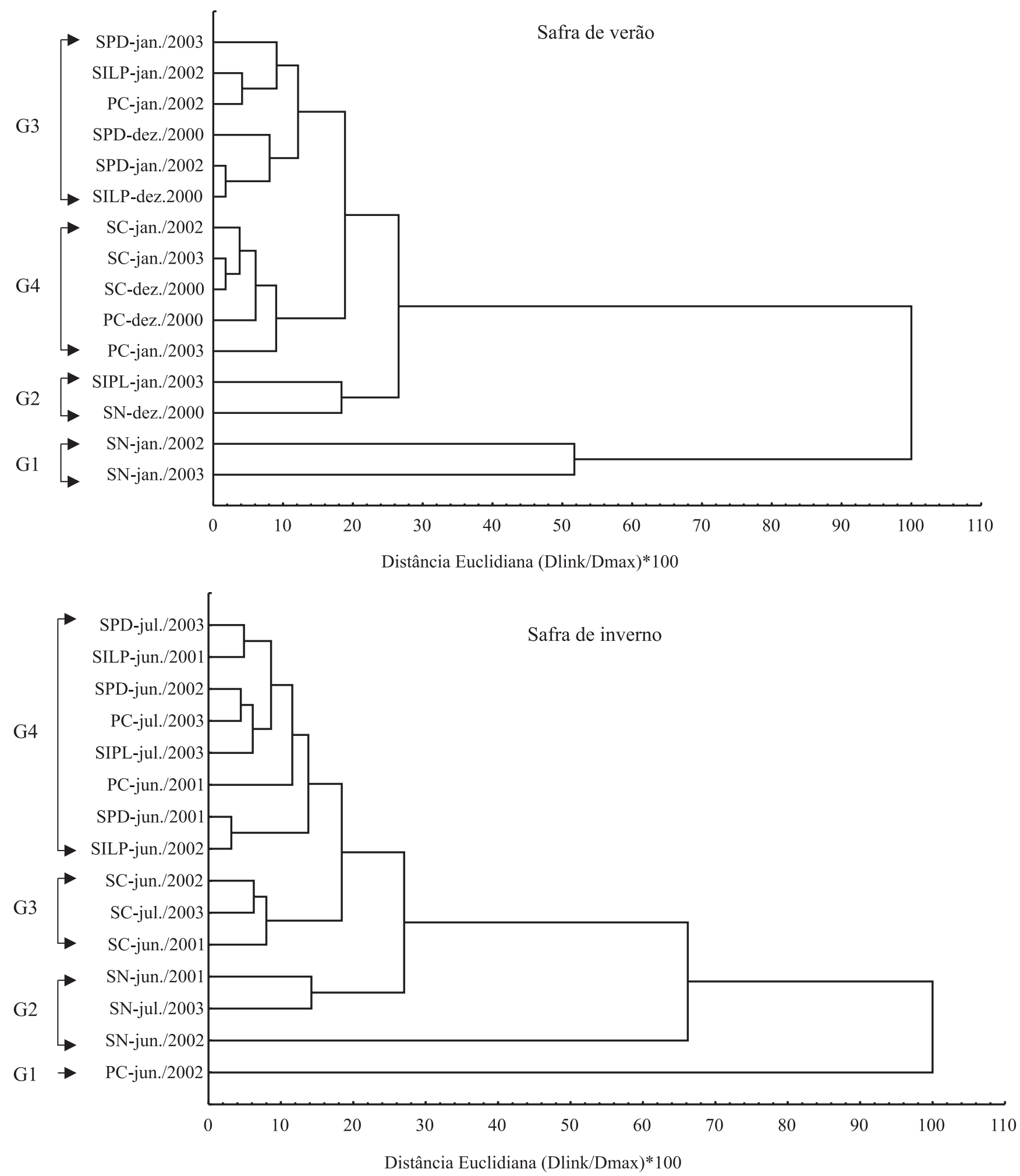

Figura 2. Dendrograma das distâncias entre as comunidades de macrofauna invertebrada de solo, nos sistemas estudados (SC: sistema convencional; SPD: sistema plantio direto; SILP: sistema integrado lavoura/pecuária; PC: pastagem contínua e SN: sistema natural sob vegetação nativa, na safra de verão (dezembro de 2000; janeiro de 2002 e janeiro de 2003) e de inverno (junho de 2001; junho de 2002 e julho de 2003). Grupos formados: G1, G2, G3 e G4. 
De modo geral, a estrutura da comunidade da macrofauna edáfica apresenta-se estável no sistema sob vegetação nativa e é menos afetada pelas práticas de manejo dos solos mais conservacionistas, como o SILP e SPD.

\section{Conclusões}

1. A densidade total da macrofauna edáfica é favorecida pelas práticas de manejo que estimulam a dinâmica da matéria orgânica do solo, como o SPD, SILP e PC.

2. A diversificação das espécies vegetais promove uma maior diversidade dos grupos da macrofauna invertebrada do solo.

3. A comunidade da macrofauna edáfica é um parâmetro sensível ao impacto de diferentes tipos de sistemas de produção, o que possibilita o seu uso como instrumento na determinação de opções de manejo sustentável dos sistemas agropecuários.

\section{Agradecimentos}

Ao CNPq, pelo apoio financeiro.

\section{Referências}

ANDERSON, J.M.; INGRAM, J.S.I. Tropical soil biology and fertility: a handbook of methods. $2^{\text {nd }}$ ed. Wallingford: CABI Pub., 1993. 240p.

BARROS, E.; NEVES, A.; BLANCHART, E.; FERNANDES, E.C.; WANDELLI, E.; LAVELLE, P. Development of the soil macrofauna community under silvopastoral and agrosilvicultural systems in Amazonia. Pedobiologia, v.47, p.273-280, 2003.

BENITO, N.P. Interferência de sistemas de cultivos sobre a macrofauna invertebrada do solo. 2002. 71p. Dissertação (Mestrado) - Universidade Estadual de Londrina, Londrina.

BENITO, N.P.; BROSSARD, M.; PASINI, A.; GUIMARÃES, M. de F.; BOBILLIER, B. Transformations of soil macroinvertebrate populations after native vegetation conversion to pasture cultivation (Brazilian Cerrado). European Journal of Soil Biology, v.40, p.147154, 2004.

BROWN, G.G. How do earthworms affect microfloral and faunal community diversity? Plant and Soil, v.170, p.209-231, 1995.

CAMPANHOLA, C. Compromissos internacionais: convenção sobre diversidade biológica. In: MANZATTO, C.V.; FREITAS JUNIOR, E.; PERES, J.R.R. (Ed.). Uso agrícola dos solos brasileiros. Rio de Janeiro: Embrapa Solos, 2002. p.135-144.

CHAN, K.Y. An overview of some tillage impacts on earthworm population abundance and diversity - implications for functioning in soils. Soil and Tillage Research, v.57, p.179-191, 2001.
CHAUSSOD, R. La qualité biologique des sols: évaluation et implications. Étude et Gestion des Sols, v.3, p.261-278, 1996.

CIVIDANES, F.J. Efeitos do sistema de plantio e da consorciação soja-milho sobre artrópodes capturados no solo. Pesquisa Agropecuária Brasileira, v.37, p.15-23, 2002.

DECÄENS, T.; LAVELLE, P.; JIMÉNEZ, J.J.; ESCOBAR, G.; RIPPSTEIN, G.; SCHNEIDMADL, J.; SANZ, J.I.; HOYOS, P.; THOMAS, R.J. Impacto del uso de la tierra en la macrofauna del suelo de los Llanos Orientales de Colombia. In: JIMÉNEZ, J.J.; THOMAS, R.J. (Ed.). El arado natural: las comunidades de macroinvertebrados del suelo en las savanas neotropicales de Colombia. Cali, Colombia: Centro Internacional de Agricultura Tropical, 2003. p.21-45. (Publicación CIAT, 336).

DIDDEN, W.A.M.; FRÜND, H.C.; GRAEFE, U. Enchytraeids. In: BENCKISER, G. (Ed.). Fauna in soil ecosystems: recycling processes nutrient fluxes, and agricultural production. New York: Marcel Dekker, 1997. p.135-172.

DIDDEN, W.A.M.; MARINISSEN, J.C.Y.; VREEKEN-BUIJS, M.J.; BURGERS, S.L.G.E.; FLUITER, R. de; GEURS, M.; BRUSSAARD, L. Soil meso and macrofauna in two agricultural systems: factors affecting population dynamics and evaluation of their role in carbon and nitrogen dynamics. Agriculture, Ecosystems and Environment, v.51, p.171-186, 1994.

EKSCHMITT, K.; WEBER, M.; WOLTERS, V. Spiders, carabids, and staphylinids: the ecological potential of predatory macroarthropods. In: BENCKISER, G. (Ed.). Fauna in soil ecosystems: recycling processes, nutrient fluxes, and agricultural production. New York: Marcel Dekker, 1997. p.307-362.

EMBRAPA. Centro de Pesquisa Agropecuária dos Cerrados (Planaltina, DF). Embrapa Cerrados e a Região dos Cerrados: informações básicas e dados estatísticos. Planaltina, DF, 1998. 24p.

EMBRAPA. Centro Nacional de Pesquisa de Solos (Rio de Janeiro, RJ). Manual de métodos de análise de solos. 2.ed. Rio de Janeiro, 1997. 212p.

KLUTHCOUSKI, J.; AIDAR, H. Uso da integração lavoura-pecuária na recuperação de pastagens degradadas. In: KLUTHCOUSKI, J.; STONE, L.F.; AIDAR, H. (Ed.). Integração lavoura-pecuária. Santo Antônio de Goiás: Embrapa Arroz e Feijão, 2003. p.185-223.

LAVELLE, P. Earthworm activities and the soil system. Biology and Fertility of Soils, v.6, p.237-251, 1988.

LAVELLE, P.; SPAIN, A.V. Soil ecology. Dordrecht: Kluwer Academic Pub., 2001. 654p.

LOBRY de BRUYN, L.A. Ants as bioindicators of soil function in rural environments. Agriculture, Ecosystems and Environment, v.74, p.425-441, 1999.

MARÍN, E.P.; FEIJOO, A.; PEÑA, J. de J. Cuantificación de la macrofauna en un vertisol bajo diferentes sistemas de manejo en el Valle del Cauca, Colombia. Revista Suelos Ecuatoriales, v.31, p.233-238, 2001.

MERLIM, A. de O.; GUERRA, J.G.M.; JUNQUEIRA, R.M.; AQUINO, A.M. de. Soil macrofauna in cover crops of figs grown under organic management. Scientia Agricola, v.62, p.57-61, 2005.

ODUM, E.P. Ecología. 3.ed. La Habana, Cuba: Edición Revolucionaria, 1989. 639p. 
OLIVEIRA, L.J.; MALAGUIDO, A.B. Flutuação e distribuição vertical da população do percevejo castanho-da-raiz, Scaptocoris castanea Perty (Hemiptera: Cydnidae), no perfil do solo em áreas produtoras de soja nas regiões centro-oeste e sudeste do Brasil. Neotropical Entomology, v.33, p.283-291, 2004.

PAOLETTI, M.G. Using bioindicators based on biodiversity to assess landscape sustainability. Agriculture, Ecosystems and Environment, v.74, p.1-18, 1999.

RÄTY, M.; HUHTA, V. Earthworms and pH affect communities of nematodes and enchytraeids in forest soil. Biology and Fertility of Soils, v.38, p.52-58, 2003.
ROBERTSON, L.N.; KETTLE, B.A.; SIMPSON, G.B. The influence of tillage practices on soil macrofauna in a semi-arid agroecosystems in Northeastern Australia. Agriculture, Ecosystems and Environment, v.48, p.149-156, 1994.

SANCHÉZ, S.; REINÉS, M. Papel de la macrofauna edafica en los ecosistemas ganaderos. Pastos y Forrajes, v.24, p.191-202, 2001.

TIAN, G.; KANG, B.T.; BRUSSAARD, L. Effect of mulch quality on earthworm activity and nutrient supply in the humid tropics. Soil Biology and Biochemistry, v.29, p.369-373, 1997.

WOLTERS, V. Invertebrate control of soil organic matter stability. Biology and Fertility of Soils, v.31, p.1-19, 2000.

Recebido em 11 de março de 2005 e aprovado em 25 de agosto de 2005 